\title{
Movilización del sentido Personal del profesor que enseña matemáticas sobre su Actividad Pedagógica desde y para las Actividades Orientadoras de Enseñanza' ${ }^{1}$
}

\author{
Mobilization of staff felt that teaches math teacher on her activity \\ to and from Pedagogical Guiding Teaching Activities
}

\begin{abstract}
Mobilização de pessoal sentiu que ensina professor de matemática em sua atividade de e para Orientadores Pedagógicos Atividades de Ensino
\end{abstract}

Recibido: mayo de 2013

Aprobado: agosto de 2013
Diego Alejandro Pérez Galeano

Diana Victoria Jaramillo Quiceno ${ }^{3}$

\section{Resumen}

Fundamentado en los presupuestos teóricos de la perspectiva histórico-cultural de la educación, y en ella la Teoría de la Actividad, planteada por Leontiev, esta ponencia presentará los análisis de un proyecto que busca analizar la movilización del sentido personal del profesor de matemáticas sobre su Actividad Pedagógica del maestro que enseña matemáticas mediada por la elaboración y socialización de Actividades Orientadoras de Enseñanza con maestros de matemáticas de la "Institución Educativa El Hatillo" del municipio de Barbosa (Antioquia).

Considerando la relación de dicha Actividad Pedagógica del maestro de matemáticas con su ideario pedagógico y la necesidad de organización de la enseñanza, propongo por medio del trabajo con los maestros una posibilidad para llevar al aula de clase los elementos construidos culturalmente.

Palabras clave: Perspectiva histórico-cultural, otras nociones de Educación Matemática; fines culturales; profesor; formación continuada de maestros; actividades orientadoras de enseñanza; enseñanza de las matemáticas; colectividad.

\section{Abstract}

Based on the theoretical assumptions of the cultural-historical perspective of education, and in it the Activity Theory, proposed by Leontiev, this paper present the analysis of a project to analyze the mobilization of personal sense math teacher about your Pedagogical Activity math teacher who teaches mediated development and socialization of Guiding Activities Teaching with math teachers "El Hatillo Educational Institution" in the municipality of Barbosa (Antioquia).

1 Artículo de Investigación.

2 Universidad de Antioquia. Medellin, Colombia. Contacto: diegoxp88@gmail.com

3 Universidad de Antioquia. Medellin, Colombia. Contacto: djaramilo.quiceno@gmail.com a 
Considering the relationship of this activity Pedagogical math teacher with pedagogical ideas and the need for organization of teaching, I propose through work with teachers a chance to bring to the classroom culturally constructed elements.

Keywords: cultural-historical perspective, other notions of Mathematics Education; cultural purposes; teacher, continuing education teachers, instructional guidance activities; teaching mathematics community.

\section{Resumo}

Com base nos pressupostos teóricos da perspectiva histórico-cultural da educação, e em que a Teoria da Atividade, proposta por Leontiev, este artigo apresenta a análise de um projeto para analisar a mobilização do professor de matemática sobre o seu sentido pessoal pedagógica professor de matemática que ensina Atividade desenvolvimento mediada e socialização de atividades de Orientação de ensino com professores de matemática "El Hatillo instituição educacional”, no município de Barbosa (Antioquia).

Considerando a relação dessa atividade professor de matemática Pedagógico com as idéias pedagógicas ea necessidade de organização do ensino, proponho através do trabalho com os professores a oportunidade de trazer para a sala de aula elementos culturalmente construídos.

Palabras clave: perspectiva histórico-cultural, outras noções de Educação Matemática; fins culturais, professor, professores de educação continuada, atividades de orientação de ensino, ensinando comunidade matemática.

\section{Planteamiento del problema}

Una de mis expectativas como docente de matemáticas es aportar desde mi propia práctica a la constitución de la subjetividad de mis estudiantes. $\mathrm{Al}$ propender por este fin, algunas inquietudes me han surgido, por ejemplo: ¿Cómo posibilitar desde las actividades que planeo la apropiación del conocimiento matemático por parte de los estudiantes? ¿Qué aspectos teóricos y metodológicos debo tener presente al momento de proponer situaciones que den cuenta de una legitimación del saber cultural e históricamente producido por la humanidad? ¿Qué comprensión de matemáticas he venido realizando desde mi proceso de formación inicial como docente y cómo dicha comprensión moviliza mi propia práctica? Estas y otras cuestiones se han constituido en uno de los motivos para plantear el proyecto en el marco de mi maestría en educación, de la cual se desprende la presente comunicación breve.
Luego de ingresar en el programa de maestría en educación, algunas ideas se estaban esclareciendo en cuanto a qué quería hacer en mi proyecto de investigación: una, formación continuada de maestros que enseñan matemáticas; $y$, dos, interés por otras formas de organizar la enseñanza en un trabajo colaborativo con los maestros. Con esto en mente, las primeras lecturas se concentraron en dos frentes: lo relacionado con el ideario pedagógico ${ }^{2}$ del maestro de matemáticas y la Teoría de la Actividad. De estas primeras lecturas realicé algunas reflexiones fundamentándome en trabajos como el de Costa (2005) y Jaramillo (2003), entre otros, en cuanto a las concepciones que el maestro tiene de sus propias actividades. Estas concepciones y creencias o, mejor, este ideario pedagógico del maestro está en dialéctica con sus formas de obrar como maestro y resultan de los movimientos históricos, sociales y culturales que cada maestro vaya sufriendo (Jaramillo, 2003).

4 El término Ideario Pedagógico es entendido en este trabajo conforme es dicho por Jaramillo (2003), como una amalgama que se refiere a las concepciones, ideas, creencias y conceptos que el maestro construye de las matemáticas, su enseñanza y su aprendizaje. 
A partir de esas lecturas y las reflexiones que me movilizaron, surgieron otras preguntas, un poco más puntuales, que quería responder con mi proyecto de maestría: ¿Qué sujeto estamos formando? ¿Cómo ven la sociedad y el propio maestro los procesos de enseñanza en la escuela? ¿Cuál es la finalidad de la educación y la escuela? ¿Qué idea de formación tiene el maestro? ¿Por qué, para qué, a quién y cómo enseñar matemáticas en la escuela? ¿Qué entiende un maestro por matemáticas? ¿Qué concepción tiene el maestro sobre su propia práctica? ¿Se forma para el "trabajo" o se "trabaja" para la formación?

De esta manera, emerge el proyecto ante las tensiones entre la significación cultural y el sentido personal del maestro que enseña matemáticas, $y$, la concepción que tiene el maestro de matemáticas frente a los fines y alcances de la enseñanza de las matemáticas en nuestro país. Considerando lo anterior, esta investigación está planteada para: uno, contribuir a la búsqueda de otras posibilidades para la organización de la enseñanza por parte de los maestros que enseñan matemáticas; dos, abrir otras posibilidades para el encuentro entre el sentido personal que tiene el maestro que enseña matemáticas frente a su Actividad Pedagógica y la significación cultural que se le ha asignado a esta; $y$, tres, para posibilitar reflexiones colaborativas por parte de los maestros en cuanto a su mirada al trabajo como una forma de actividad exclusivamente humana que media en la constitución de la humanidad y no como fin de la educación.

Dado todo lo anterior, el proyecto en marcha tiene como pregunta desencadenadora ¿Cómo se da la movilización del Sentido Personal del profesor que enseña matemáticas de su Actividad Pedagógica desde y para las Actividades Orientadoras de Enseñanza? En coherencia, el objetivo es Analizar la movilización del Sentido Personal del profesor que enseña matemáticas de su Actividad Pedagógica desde y para las Actividades Orientadoras de Enseñanza.

\section{Horizonte teórico}

Tres son los pilares que sustentan el marco teórico de la presente propuesta investigativa: la Teoría de la Actividad, desde una perspectiva histórico-cultural de la educación; la Actividad Pedagógica como forma particular de Actividad en el ámbito educativo, y en ella la Formación Continuada de Maestros que enseñan matemáticas; y las Actividades Orientadoras de Enseñanza como posibilidad desde lo teórico y metodológico para la significación de la Actividad Pedagógica. A continuación presentaré algunos autores que me han guiado en este constructo teórico, los cuales servirán en el momento del análisis para la triangulación con los datos obtenidos junto con mi mirada investigativa.

En este primer apartado del marco teórico intentaré aproximarme de estos elementos. La actividad se refiere a las formas de organización al interior de una cultura, orientadas a un fin determinado. Parafraseando a Davidov (1988) considero que la actividad no puede verse, entonces, como una reunión de personas que se encuentran para resolver una tarea; una actividad, en el sentido de Leontiev debe considerarse como una secuencia interconectada de acciones a través de las cuales los individuos se relacionan no solamente con el mundo de los objetos sino también con otros individuos, adquiriendo, en el curso de ese proceso, la experiencia humana. En este mismo apartado del horizonte teórico, destaco a Asbahr (2005), quien ha trabajado concentrando su atención sobre el Sentido Personal en el Ámbito de la Actividad, Moretti (2007), quien aborda la Actividad en el contexto de formación continuada de maestros, entre otros.

En cuanto a la Actividad Pedagógica, es la unidad dialéctica que se establece entre las dos actividades presentes en el aula de clase: la Actividad de Enseñanza del maestro y la Actividad de Aprendizaje del estudiante; y ella se consolida como elemento central al momento de referirse a la actividad propia del contexto educativo. Como afirman Rigon, Bernardes, Moretti \& Cedro: 
En lo que se refiere al desarrollo humano vinculado al proceso educativo, se debe dar énfasis al estudio de la Actividad Pedagógica, como unidad dialéctica entre la enseñanza y el aprendizaje, mediada por los significados sociales elaborados por el hombre y constituyentes de los individuos que tienen acceso al conocimiento elaborado históricamente. (2010, p. 65)

Otros autores referenciados para esta Actividad Pedagógica son Rosa, Moraes \& Cedro (2010); Moura et al (2010), entre otros.

Finalmente, según Moura et al. (2010) una Actividad Orientadora de Enseñanza es una actividad que se estructura de modo que permita que los sujetos interactúen, mediados por un contenido negociando significados, con el objetivo de solucionar colectivamente una situación presente en su contexto. La Actividades Orientadoras de Enseñanza se constituye en una unidad entre la Actividad de Enseñanza y la Actividad de Aprendizaje pues surge como una posibilidad para poner en diálogo las acciones de maestros y estudiantes para legitimar colectivamente un saber específico.

\section{Metodología}

El camino a recorrer en esta investigación estará orientando mis formas de actuar, de realizar el trabajo de campo y el posterior análisis.

En primer lugar, este proyecto está planteado como una investigación de tipo cualitativo. Este paradigma se caracteriza por analizar al ser humano en todas sus dimensiones, reconociendo desde su subjetividad sus aspectos sociales, políticos, culturales, entre otros (Denzin, Guba, \& Lincoln, 1994). Al tener un horizonte cualitativo, se favorece el análisis de una significación de la Actividad Pedagógica de los maestros, ya que al plantear los instrumentos de producción de registros, una mirada cualitativa daría cuenta del movimiento en su forma natural, sin estandarizar ni plantear hipótesis a priori frente al comportamiento de los sujetos.
En segundo lugar, al interior de la investigación cualitativa, el enfoque crítico-dialéctico también enmarca los análisis ya que esta manera de concebir la investigación posibilita una transformación en los sujetos en sus contextos socio-históricos. Al respecto, Sánchez (1998) argumenta que el abordaje crítico-dialéctico

Presenta entre sus presupuestos filosóficos diversas concepciones de hombre que pueden ser resumidas así: el hombre es considerado en la mayoría de las investigaciones como un ser social, es decir, individuo inserto en el conjunto de las relaciones sociales. A pesar de ser histórica y socialmente determinado también es capaz de tomar conciencia de su papel histórico. (p. 85)

Los maestros que serán protagonistas de la investigación y que conformarán con el investigador el grupo colaborativo pertenecen al área de matemáticas de la "Institución Educativa El Hatillo" del municipio de Barbosa (Antioquia). Son nueve los maestros del área, que trabajan desde la coordinación de la maestra Luz Adriana Cadavid Muñoz. La maestra es estudiante del programa de Doctorado en Educación de la Universidad de Antioquia y es con quien se ha acordado, luego de su diálogo con dichos maestros, la realización del trabajo de campo. Algunos instrumentos que serán utilizados para la producción de registros son las mismas Actividades Orientadoras de Enseñanza que los maestros diseñan, sus discursos, ideogramas, autobiografías, entre otros. El análisis se llevará a cabo con una triangulación entre los datos producidos, los referentes teóricos y mi mirada como investigador. Las principales unidades de análisis son los enunciados de los maestros, los cuales, entre otras cosas, me permitirán ver el movimiento del sentido personal que ellos vayan constituyendo en dialéctica con los otros. En la ponencia se presentarán los análisis de los datos producidos y el camino a las categorías de análisis, junto con las conclusiones derivadas tanto del objetivo propuesto como del análisis efectuado 


\section{Referencias}

Asbahr, F. (2005a). Sentido pessoal e Projeto político pedagógico: análise da atividade pedagógica a partir da psicologia histórico-cultural. Recuperado el 01 de 04 de 2012, de http://www.teses.usp.br/teses/disponiveis/47/47131/tde.../DissertFlavia1.pdf

Cadavid, L. A., \& Quintero, C. P. (2011). Función: proceso de objetivación y subjetivación en clase de matemáticas (Tesis de Maestría no publicada). Universidad de Antioquia. Medellín.

Cedro, W. L. (2008). O motivo e a atividade de aprendizagem do professor de Matemática: uma perspetiva histórico-cultural (Tesis de Doctorado no publicada). Universidad de Sao Paulo. Brasil.

Costa, R. (2005). Concepções e crenças de professores de matemática. Recuperado el 01 de 04 de 2012, de http://www.pucpr.br/eventos/educere/educere2005/anaisEvento/documentos/ painel/TCCI254.pdf

Davídov, V. (1988). La enseñanza escolar y el desarrollo psíquico. (M. Shuare, Trad.) Moscu: Progreso.

Denzin, N., Guba, E. \& Lincoln, Y. (1994). Competing paradigms in qualitative research. Handbook of qualitativeresearch. Park, CA: SAGE Publications.

Jaramillo, D. (2003). (Re)constituição do ideário de futuros professores de Matemática num contexto de investigação sobre a prática pedagógica. (Tesis de doctorado no publicada). Universidade Estadual de Campinas, Campinas.

Moretti, V. (2007). Professores de matemática em atividade de ensino. Uma perspectiva histórico-cultural para a formação docente. (Tesis de doctorado). Recuperado el 01 de 04 de 2012, de http://www.teses.usp.br/teses/disponiveis/48/48134/tde-05102007-153534/pt-br. php

Moura, M. O., Araujo, E. S., Ribeiro, F. D., Panossian, M. L., \& Moretti, V. D. (2010). A Atividade Orientadora de Ensino como Unidade entre Ensino e Aprendizagem. En M. O. Moura, A atividade pedagógica na teoría HistóricoCultural (pp. 81-109). Brasilia: Liber Livro.

Rigon, A. J., Bernardes, M. E., Moretti, V. D., \& Cedro, W. L. (2010). O Desenvolvimento Psíquico e o Processo Educativo. En M. O. Moura, A atividade pedagógica na teoria Histórico-Cultural (pp. 45-66). Brasilia: Liber Livro.

Rosa, J. E., Moraes, S. P., \& Cedro , W. L. (2010). As Particularidades del Pensamento Empírico e do Pensamento Teórico na organização do Ensino. En M. O. Moura, A atividade pedagógica na teoria Histórico-Cultural (pp. 67-80). Brasilia: Liber Livro.

Sánchez, S. (1998). Fundamentos para la investigación educativa. Presupuestos epistemológicos que orientan al investigador. Bogotá: Cooperativa Editorial Magisterio 